\title{
Cluster Monte Carlo simulation of the transverse Ising model
}

\author{
Henk W. J. Blöte ${ }^{1,2}$ and Youjin Deng ${ }^{1}$ \\ ${ }^{1}$ Faculty of Applied Sciences, Delft University of Technology, P.O. Box 5046, 2600 GA Delft, The Netherlands \\ ${ }^{2}$ Lorentz, Institute, Leiden University, P.O. Box 9506, 2300 RA Leiden, The Netherlands
}

(Received 17 July 2002; published 10 December 2002)

\begin{abstract}
We formulate a cluster Monte Carlo method for the anisotropic limit of Ising models on $(d+1)$-dimensional lattices, which in effect, are equivalent with $d$-dimensional quantum transverse Ising models. Using this technique, we investigate the transverse Ising models on the square, triangular, Kagome, honeycomb, and simple-cubic lattices. The Monte Carlo data are analyzed by finite-size scaling. In each case we find, as expected, that the critical behavior fits well in the $(d+1)$-dimensional Ising universality class. For the transverse Ising model on the square lattice, we determine the Binder cumulant of the classical counterpart for a range of aspect ratios between the system sizes in the third or "classical" direction and that in the other two directions. Matching this universal function with the case of the isotropic Ising model yields the length ratio relating the isotropic Ising model with the anisotropic limit. The efficiency of the present algorithm is reflected by the precision of its results, which improves significantly on earlier analyses.
\end{abstract}

DOI: 10.1103/PhysRevE.66.066110 PACS number(s): 05.50.+q, 64.60.Cn, 64.60.Fr, 75.10.Hk

\section{INTRODUCTION}

It is well known that the $d$-dimensional quantum transverse Ising model (TIM) is equivalent with the anisotropic limit of $(d+1)$-dimensional lattice Ising model. As early as in 1964, Schultz and Mattis [1] displayed this equivalence by mapping the classical Ising model on a quantum model that reduces to the TIM. The reverse path, i.e., from quantum spin models to anisotropic lattice models, was shown by Suzuki $[2,3]$, by using the Trotter formula [4].

This equivalence enables one to explore the properties of the TIM by the study of its classical counterpart. In this way, one can take advantage of the insight and results that have been obtained from the theory of classical critical phenomena, including the renormalization theory. For instance, one can study the TIM in any number of dimensions, by means of the discretized path integral approach $[5,6]$, or by applying standard Monte Carlo techniques to its classical counterpart [7].

However, these numerical techniques lead to practical difficulties due to singular behavior in the anisotropic limit of the classical Ising model. When this Hamiltonian limit is approached, the coupling strengths and the correlation length in one of the directions in the classical model diverge, while the couplings in the other directions approach zero. Possibilities to deal with this problem are to approximate the anisotropic limit by a properly strong anisotropy, or extrapolate by taking the anisotropy stronger and stronger [7]. Such simulations tend to be difficult as a consequence of the considerable requirements of computer time and memory.

In this paper, we tackle this problem by means of the direct application of a continuous cluster algorithm in the anisotropic limit of the Ising model. As the correlation length in the strong-coupling direction diverges when the Hamiltonian limit is approached, we increase the number of spins in this direction and meanwhile rescale it by a divergent number such that the physical size of the system remains constant. This rescaling renders the strong-coupling dimension continuous, while the other dimensions remain discrete.
Thus, there is an infinite number of spins per physical length unit along the strong-coupling direction. In this continuous limit, cluster algorithms can be formulated [8] whose efficiencies are comparable to the conventional cluster methods for the isotropic case.

The precision of the results obtained by this continuous algorithm indicates that it is efficient in comparison with other methods that have been used to investigate transverse Ising models. These results are in agreement with the expectation that quantum transverse Ising models belong to the universality class of the classical Ising model with one more dimension. An interesting property of this continuous cluster algorithm is that it can be applied to systems in curved geometries [9].

The outline of this work is as follows. In Sec. II, we review the anisotropic limit of the $(d+1)$-dimensional Ising model and its equivalence to the $d$-dimensional TIM, while keeping track of the divergent length scale in $(d+1)$ st direction. Section III describes the continuous cluster algorithm. In Sec. IV, we investigate the $d=1$ TIM as a test case, and address the efficiency of the algorithm. Section $\mathrm{V}$ presents applications to several $d=2$ TIM's, and to the $d=3$ TIM on the simple-cubic lattice.

\section{THE ANISOTROPIC LIMIT OF THE ISING MODEL}

Using $d=1$ as an example, we briefly recall the relation between the $(d+1)$-dimensional classical Ising model and the $d$-dimensional TIM. In view of its relevance for Monte Carlo analyses, we put some emphasis on the behavior of the length scale in the $(d+1)$ st direction. The Hamiltonian of a classical two-dimensional Ising model on a $N \times M$ square lattice with periodic boundary conditions is defined by

$$
\mathcal{H} / k_{\mathrm{B}} T=-\sum_{x, y}\left[K_{x} s_{x, y} s_{x+1, y}+K_{y} s_{x, y} s_{x, y+1}\right]
$$

where the integer coordinates $x$ and $y$, which are defined modulo $N$ and $M$, respectively, label the lattice sites, and $K_{x}$, 
$K_{y}$ are the coupling strengths in the $x$ and $y$ directions, respectively. The spins can assume the values $s_{x, y}= \pm 1$. The critical line of this model is given by [10]

$$
\sinh \left(2 K_{x}\right) \sinh \left(2 K_{y}\right)=1 .
$$

Therefore, in the anisotropic limit $\epsilon \rightarrow 0$, the couplings can be written as

$$
K_{x}=\epsilon / t, \quad \exp \left(-2 K_{y}\right)=\epsilon,
$$

where $t$ parametrizes the temperature; the critical point is $t_{c}$ $=1$.

The evaluation of the partition function $Z(M, N)$ of this model by means of the transfer matrix $\mathbf{T}$ is expressed by

$$
Z(M, N)=\sum_{\vec{s}_{1}, \vec{s}_{2}, \ldots, \vec{s}_{M}}\left\langle\vec{s}_{1}|\mathbf{T}| \vec{s}_{2}\right\rangle\left\langle\vec{s}_{2}|\mathbf{T}| \vec{s}_{3}\right\rangle \cdots\left\langle\vec{s}_{M}|\mathbf{T}| \vec{s}_{1}\right\rangle,
$$

where the transfer direction is taken along the strong bonds $K_{y}$, and the elements of $\mathbf{T}$ are

$$
\left\langle\vec{s}_{k}|\mathbf{T}| \vec{s}_{k+1}\right\rangle=\prod_{i} \exp \left[K_{x} s_{x, y} s_{x+1, y}+K_{y} s_{x, y} s_{x, y+1}\right]
$$

Here $\vec{s}_{k}$ and $\vec{s}_{k+1}$ are the spin configurations in two adjacent rows, respectively. Equation (4) is just the trace of $\mathbf{T}^{M}$, so that the partition function is the sum over the $M$ th powers of the eigenvalues of $\mathbf{T}$. For large $M$, the contribution from the largest eigenvalue dominates. Since every different spin contributes a factor $\epsilon, \vec{s}_{k}$ and $\vec{s}_{k+1}$ must be nearly identical. Thus, up to order $\epsilon$ we may represent the transfer matrix as

$$
\left\langle\vec{s}_{k}|\mathbf{T}| \vec{s}_{k+1}\right\rangle=\left\langle\vec{s}_{k}\left|\exp \left(-\frac{\epsilon}{t} \mathcal{H}_{\mathrm{qm}}\right)\right| \vec{s}_{k+1}\right\rangle \exp \left(N K_{y}\right),
$$

in which $\mathcal{H}_{\mathrm{qm}}$ is the one-dimensional quantum Hamiltonian

$$
\mathcal{H}_{\mathrm{qm}}=-\sum_{x}\left(s_{x}^{z} s_{x+1}^{z}+t s_{x}^{x}\right)
$$

where $s^{z}$ and $s^{x}$ are Pauli matrices. $\mathcal{H}_{\mathrm{qm}}$ contains noncommuting operators and represents a quantum system with Ising interactions between the nearest-neighboring spins along the chain, and a transverse field $t$ in the $x$ direction. This establishes the relation between the two-dimensional Ising model and the one-dimensional TIM.

As mentioned earlier, one can also derive this equivalence by using the Trotter formula [4], which can be written as

$$
\exp \left[-\beta \mathcal{H}_{\mathrm{qm}}\right]=\lim _{M \rightarrow \infty}\left\{\exp \left[-\frac{\beta}{M} \mathcal{H}_{\mathrm{qm}}\right]\right\}^{M},
$$

where $\beta$ is the inverse temperature of the quantum system.

A comparison of Eqs. (6) and (8) yields the relation between the inverse temperature $\beta$ of the TIM and the lattice size $M$ for the classical Ising model as

$$
M=\beta t / \epsilon .
$$

The equivalence of the TIM and the classical model thus requires that $M$ diverges as $1 / \epsilon$ even at nonzero temperatures. This is a serious complication for simulations, especially at low quantum temperatures $\beta \rightarrow \infty$.

For $d \geqslant 2$, we use the example of the Ising model on the simple-cubic lattice. Its Hamiltonian is

$$
\begin{aligned}
\mathcal{H} / k_{\mathrm{B}} T= & -\sum_{x=1}^{N} \sum_{y=1}^{N} \sum_{z=1}^{M_{\mathrm{p}}}\left[K_{x y} s_{x, y, z}\left(s_{x+1, y, z}+s_{x, y+1, z}\right)\right. \\
& \left.+K_{z} s_{x, y, z} s_{x, y, z+1}\right],
\end{aligned}
$$

where $1 \leqslant x, y \leqslant N$, and $1 \leqslant z \leqslant M_{p}$ label the lattice sites. The label $p$ emphasizes that $M_{p}$ refers to the physical system size; its ratio with $N$ defines the aspect ratio of the threedimensional system. Periodic boundary conditions apply. The coupling strengths $K_{x y}$ and $K_{z}$ in the $x y$ plane and in the $z$ direction, respectively, are initially chosen to be of order 1 . The behavior of the length scales in the Hamiltonian limit, where $K_{z}$ diverges while $K_{x y}$ approaches zero, is illustrated by means of a Migdal-type renormalization [11] in the $z$ direction, without rescaling the $x$ and $y$ directions. We expect that this procedure, although only valid as an approximation, will yield a qualitatively correct picture. Each bond in the $z$ direction is decorated with $n-1$ Ising spins and the bond strength $K_{x y}$ is distributed accordingly among the newly inserted spins. This leads to a model with a lattice spacing along the $z$ direction which is smaller by a factor $n$. It is described by the same Hamiltonian Eq. (10) but with new couplings $K_{x y}^{(n)}$ and $K_{z}^{(n)}$, and the $z$ coordinate is represented by integers $z^{\prime}=n z$ which run from 1 to $M^{\prime}=n M_{p}$,

$$
\begin{aligned}
\mathcal{H}^{\prime} / k_{\mathrm{B}} T= & -\sum_{x=1}^{N} \sum_{y=1}^{N} \sum_{z^{\prime}=1}^{M^{\prime}}\left[K_{x y}^{(n)} s_{x, y, z^{\prime}}\left(s_{x+1, y, z^{\prime}}+s_{x, y+1, z^{\prime}}\right)\right. \\
& \left.+K_{z}^{(n)} s_{x, y, z^{\prime}} s_{x, y, z^{\prime}+1}\right] .
\end{aligned}
$$

The new couplings satisfy

$$
K_{x y}^{(n)}=K_{x y} / n \quad \text { and } \quad \tanh K_{z}^{(n)}=\left[\tanh K_{z}\right]^{1 / n} .
$$

For large $n$, one may write $\exp \left(-2 K_{z}^{(n)}\right)=\epsilon$, and substitute $\tanh K_{z}^{(n)}$ in Eq. (12) accordingly. One finds $1 / n=a[\ln (1$ $+\epsilon)-\ln (1-\epsilon)] \simeq a \epsilon\left[2+O\left(\epsilon^{2}\right)\right]$, where $a=-1 / \ln \left(\tanh K_{z}\right)$. This leads to

$$
K_{x y}^{(\infty)}=\frac{\epsilon}{t}\left[1+O\left(\epsilon^{2}\right)\right], \quad \exp \left[-2 K_{z}^{(\infty)}\right]=\epsilon,
$$

which has a same form as Eq. (3). It also suggests that for nonzero $\epsilon$ the critical point $t_{c}(\epsilon)$ deviates from $t_{c}(0)$ as $\epsilon^{2}$.

This model is equivalent with the TIM on the square lattice with Hamiltonian

$$
\mathcal{H}_{\mathrm{qm}}=-\sum_{x, y}\left[s_{x, y}^{z}\left(s_{x+1, y}^{z}+s_{x, y+1}^{z}\right)+t s_{x, y}^{x}\right]
$$


The inverse proportionality of $n$ and $\epsilon$, together with Eq. (9), shows that the physical size $M_{p}=M^{\prime} / n$ is proportional to the inverse quantum temperature $\beta$.

\section{ALGORITHM}

As mentioned earlier, a Monte Carlo method for the Hamiltonian limit will have to deal with singular aspects such as the divergent coupling strength $K_{z}$, the vanishing coupling strength $K_{x y}$, and the divergence of the system size $M$. Using ideas from existing cluster methods [12-14], we introduce procedures to improve the efficiency for the system described by Eqs. (1) and (3) with small but nonzero $\epsilon$. Then we discuss how to deal with the divergence of the system size $M$ in the $y$ direction, and finally describe the continuous Wolff algorithm (CWA) for the limit $\epsilon=0$.

Now, let us recall the cluster algorithm for the isotropic lattice Ising model with nearest-neighbor interactions. If two nearest-neighboring spins on sites $m$ and $n$, coupled with strength $K_{m n}$, have the same sign the algorithm will "freeze" the bond between $m$ and $n$ with a probability $p_{m n}$ $=\left[1-\exp \left(-2 K_{m n}\right)\right]$, or "break" the bond with the probability $1-p_{m n}$. Sites connected by "frozen" bonds are included in the same cluster. One can introduce bond variables $b_{m n}$ $=0$ or 1 ; frozen bonds have $b_{m n}=1$ and broken bonds have $b_{m n}=0$. A pair of opposite spins always has $b_{m n}=0$. The conventional way to simulate this is to draw a uniformly distributed random number $r(0<r<1)$ for each bond $b_{m n}$, set $b_{m n}=1$ if the spins on sites $m$ and $n$ have the same sign and $r<p_{m n}$.

For the anisotropic model defined by Eqs. (1) and (3), there are two types of bond variables $b_{m n}$. For small $\epsilon$, the bond probability between a pair of equal spins in the $y$ direction is $p_{y}=1-\exp \left(-2 K_{y}\right) \propto 1-\epsilon$, so one has to draw of order $1 / \epsilon$ random numbers $r$ before finding a bond variable $b_{y}=0$. For the weak bonds in the $x$ direction, the probability $p_{x}=1-\exp \left(-2 K_{x}\right) \propto \epsilon$ that a pair of equal neighbors is connected by a frozen bond is small, and many random numbers are needed before such a "bridge" is found.

A more efficient procedure follows. We first write $b_{m n}$ $=\widetilde{b}_{m n} \delta_{s_{m} s_{n}}$, where the $\widetilde{b}_{m n}$ are independent random variables equal to 0 or $1 ; \widetilde{b}_{m n}=1$ with probability $p_{x}=1-\exp$ $\left(-2 K_{x}\right)$ or $p_{y}=1-\exp \left(-2 K_{y}\right)$ for bonds in the $x$ or $y$ direction, respectively. Counting the bond variables sequentially in the $y$ direction, the distribution $P_{y}\left(k_{y}\right) \equiv\left(1-p_{y}\right) p_{y}^{k_{y}-1}$ expresses the probability that $\left(k_{y}-1\right)$ subsequent bond variables $\widetilde{b}_{m n}$ are equal to 1 , while the $k_{y}$ th variable is zero: a break occurs at the $k_{y}$ th position. Thus the cumulative distribution is

$$
C_{y}\left(k_{y}\right)=\sum_{k=1}^{k_{y}} P_{y}(k)=1-\left(p_{y}\right)^{k_{y}}
$$

and by mapping the probability distribution $C_{y}\left(k_{y}\right)$ on the uniform distribution of the random number $r$, one can transform $r$ into an integer $k_{y}$,

$$
k_{y}=1+\left[\ln (r) / \ln \left(p_{y}\right)\right]
$$

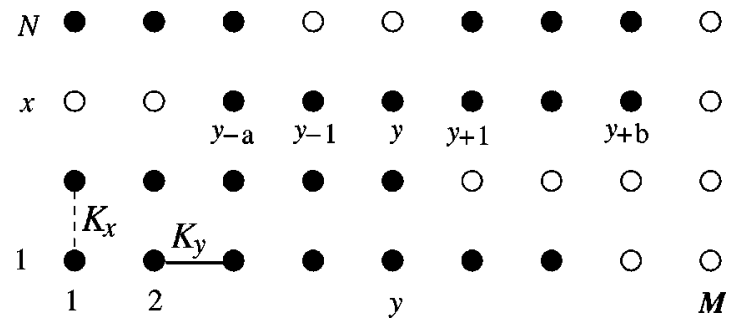

FIG. 1. Illustration of the anisotropic Ising model on an $N \times M$ lattice. The vertical dashed line represents the weak coupling $K_{x}$; the horizontal line represents the strong coupling $K_{y}$. The black circles are + spins, and the open circles are - pins.

where $0<r<1$ and the square brackets denote the integer part. In contrast, in the $x$ direction, one uses the distribution $P_{x}\left(k_{x}\right) \equiv p\left(1-p_{x}\right)^{k_{x}-1}$ to express the probability that $\left(k_{x}\right.$ $-1)$ subsequent variables $\widetilde{b}_{m n}$ are zero, while the $k_{x}$ th bond variable is 1 . Also in this case, one transforms a uniformly distributed random number $r$ into an integer $k_{x}$,

$$
k_{x}=1+\left[\ln (r) / \ln \left(1-p_{x}\right)\right] .
$$

This method avoids the problem that many random numbers have to be drawn before adding a new bridge or a new break.

For purpose of clarity, we describe in detail the steps in the formation of a Wolff cluster according to the description above.

(1) Choose the origin $(x, y)$ of the cluster randomly (see Fig. 1), denote its sign $s \equiv s_{x, y}$.

(2) Count the neighboring sites on the left-hand side as $(x, y-1),(x, y-2), \ldots,(x, y-a)$ till the nearest interface $s_{x, y-a-1}=-s$ (see Fig. 1). Draw a random number $r$ and compute $k_{y}$ according to Eq. (16). Define $l_{-}$as the smaller number of $a$ and $k_{y}-1$, and flip the spins from $\left(x, y-l_{-}\right.$ $+1)$ to $(x, y)$. Do the same for the right-hand side such that the spins from $(x, y+1)$ to $\left(x, y+l_{+}\right)$are flipped. Thus, a range of $l_{-}+l_{+}$strongly coupled spins on the $x$ th row is included in the cluster and flipped.

(3) Include into the cluster spins on $(x-1)$ th and $(x$ $+1)$ th rows connected to the above range by bridges in the weak-coupling direction. Compute $k_{x}$ according to Eq. (17). If $k_{x}>l_{-}+l_{+}$, go to (4). Otherwise, if $s_{x-1, y-l_{-}+k_{x}}=s$, include this spin in the cluster and store its position in the "stack" memory. Find a new random value $k_{x}$ till all the $l_{-}+l_{+}$bonds between $x$ th and $(x-1)$ th rows are accounted for. Do the same for the neighboring sites on the $(x+1)$ th row.

(4) If the stack is empty, go to (5). Otherwise, read a site $(x, y)$ from the stack and erase it from the stack. Go to (2).

(5) The cluster is completed and flipped.

Although the above procedures can improve the efficiency of the conventional Wolff algorithm, we still have to solve the problem of the divergence of the expectation values of $k_{x}$ and $k_{y}$ in parallel with that of $M$ as $\epsilon \rightarrow 0$,

$$
\left\langle k_{y}\right\rangle=\frac{-1}{\ln \left(p_{y}\right)} \propto \frac{1}{\epsilon},\left\langle k_{x}\right\rangle=\frac{-1}{\ln \left(1-p_{x}\right)} \propto \frac{1}{\epsilon} .
$$




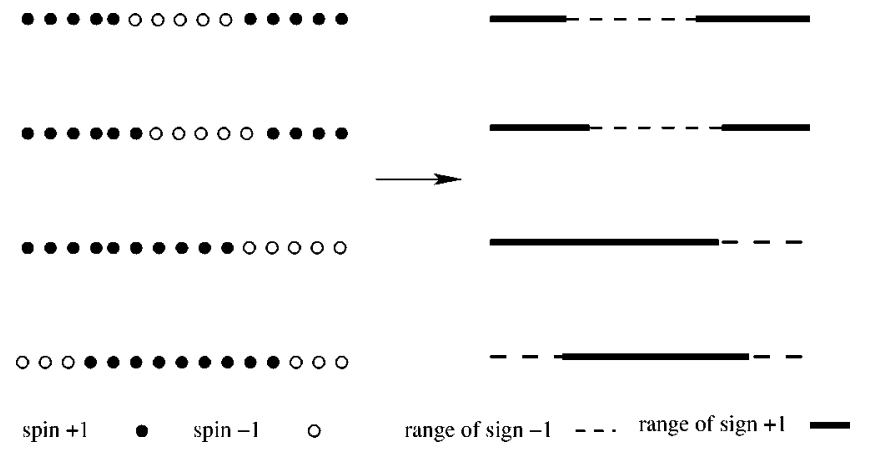

FIG. 2. Illustration of the procedure leading to the Hamiltonian limit of the classical Ising model. The physical length scale is approximately conserved by reducing the horizontal size of $N \times M / \epsilon$ spins with a factor $\epsilon$. This leads to $N$ continuous lines of length $M$ in the Hamiltonian limit. The left figure shows an anisotropic Ising model with small but nonzero $\epsilon$, and the right one illustrates the $\epsilon=0$ case.

$\left\langle k_{y}\right\rangle$ and $\left\langle k_{x}\right\rangle$ can be recognized as the average distances, in the $y$ direction, of the breaks and of the bridges, respectively.

To deal with the divergence of the system size $M$ one can rescale the $y$ direction as $y_{p}=\epsilon y$, so that the total physical size $M_{p}=\epsilon M$ and the correlation length in this direction remain approximately constant (see Fig. 2). In the limit $\epsilon$ $=0$, the strong-coupling dimension becomes continuous, i.e., there are infinite number of spins per physical length unit, and the $+I-$ spins are replaced by ranges of sign $+/-$. Thus, the $N \times M$ square lattice reduces to $N$ lines of physical length $M_{p}=\epsilon M$ (see Fig. 2), and Eqs. (16) and (17) change into

$$
l_{y}=\epsilon k_{y}=-\ln (r)
$$

and

$$
l_{x}=\epsilon k_{x}=-\ln (r) t / 2
$$

which indicate that the breaks and the bridges occur on a length scale of 1 so that the numbers of these breaks and bridges are finite in this continuous limit. These breaks can be recognized as interfaces separating the ranges of + and - spins, and the bridges serve as the connections between nearest-neighboring lines. Instead of the individual spins one may use the locations of these interfaces as the dynamical variables, and define a CWA on this basis.

In the conventional Wolff algorithm, the spins are simply stored in an array. Since this information is no longer available, one has to determine the sign of a particular position $(x, y)$ from the positions of the interfaces, supplemented with additional information on the sign at a given position on each continuous line, e.g., at the origin $y=0$. The sign at position $(x, y)$ is given by $s_{x}(-1)^{n}$, where $n$ is the number of interfaces between the origin and position $y$ on the $x$ th line, and $s_{x}$ is the sign at the origin of this line.

The steps involved in the CWA can now be expressed as follows:
(1) Choose a random position $(x, y)$ randomly, which means it is at $x$ th line and its $y$ coordinate is $y$, and obtain its sign $s$ according to the directions given above.

(2) Determine the distance $d_{l}$ from $y$ to the first interface on the left-hand side of $y$, and similarly the distance $d_{r}$ on the right-hand side.

(3) Include a range around $(x, y)$ into the cluster as follows. Draw a random number $r$ and obtain $l_{y}$ from Eq. (19). If $l_{y}<d_{l}$, create an interface at position $\left(x, y-l_{y}\right)$; otherwise, annihilate the interface at $\left(x, y-d_{l}\right)$. So the left-hand end of the range to be flipped is set at $\left(x, y-c_{l}\right)$, where $c_{l}$ is the smaller number of $d_{l}$ and $l_{y}\left[c_{l}=\min \left(d_{l}, l_{y}\right)\right]$. Find another number from Eq. (19) and obtain the right-hand end of the range $\left(x, y+c_{r}\right)$ analogously. Thus, the range from $(x, y$ $\left.-c_{l}\right)$ to $\left(x, y+c_{r}\right)$ is included in the cluster and flipped.

(4) Create bridges between this range and its nearestneighboring lines. For the $(x-1)$ th line, draw a random number and compute $l_{x}$ by Eq. (20). If $l_{x}>c_{l}+c_{r}$, go to (5). Otherwise, if the sign at position $\left(x-1, y-c_{l}+l_{x}\right)$ is equal to $s$, store the position into the stack. Repeat this procedure till the whole range has been visited. Do the same for the $(x$ +1 )th line.

(5) If the stack is empty, go to 6. Otherwise, read $(x, y)$ from the stack, and erase it from the stack. Go to (2).

(6) The cluster is completed and flipped.

In the CWA a spin range is flipped by the creation or annihilation of interfaces. When a range is flipped, there are three possibilities: two interfaces are created, two interfaces are annihilated, or one new interface is created and an existing one is annihilated. In all these cases, the number of interfaces per line remains even. One detail to be mentioned is that, if a flipped range includes the origin of that line, the corresponding array element containing the signs at the origins, should be changed.

It is straightforward to generalize the CWA for applications to $d$-dimensional TIM's with $d \geqslant 2$. Related continuous cluster algorithms, such as the Swendsen-Wang variety, can also trivially be formulated on the basis of the above description.

\section{TEST OF THE ALGORITHM}

To test the CWA, we investigated the anisotropic limit of the two-dimensional Ising model defined by Eqs. (1) and (3), since it has been solved exactly [10]. The CWA was applied to simulate such systems with $L$ lines of length $L$ and with periodic boundary conditions, where $L=8,12,16,22$, and 24 . During the simulations, the dimensionless quantity $Q_{L}$, which is related to the Binder cumulant [15], was sampled,

$$
Q_{L}(t)=\frac{\left\langle m^{2}\right\rangle_{L}^{2}}{\left\langle m^{4}\right\rangle_{L}},
$$

where $m$ is the magnetization density.

According to universality of the Binder cumulant, the asymptotic value of $Q$ in such a system is equal to that for the lattice Ising model, with isotropic couplings $K_{x}=K_{y}$ and system sizes $L \times \alpha L$, where $\alpha=\lim _{\epsilon \rightarrow 0} 1 /\left(\epsilon \sinh 2 K_{y}\right)=2$ $[10,16]$. 
TABLE I. Results of the least-squares fits of $Q_{L}(t)$ for the TIM's defined on the triangular, Kagome, honeycomb, square, and cubic lattices.

\begin{tabular}{llllll}
\hline \hline & Triangular & Kagome & Honeycomb & Square & Cubic \\
\hline$L_{\min }$ & 6 & 8 & 10 & 2 & 7 \\
$L_{\max }$ & 20 & 20 & 20 & 48 & 14 \\
$Q$ & $0.6238(7)$ & $0.6041(4)$ & $0.6149(7)$ & $0.6206(2)$ & 0.456947 (fixed) \\
$t_{c}$ & $4.76811(9)$ & $2.95265(4)$ & $2.13250(4)$ & $3.04438(2)$ & $5.15813(6)$ \\
$a_{1}$ & $0.03138(5)$ & $0.0894(1)$ & $0.1027(8)$ & $0.0497(2)$ & $0.0235(2)$ \\
$a_{2}$ & $0.0010(1)$ & $0.0082(2)$ & $0.0088(2)$ & $0.00207(2)$ & $0.0020(4)$ \\
$a_{3}$ & $-0.00023(7)$ & $-0.0035(5)$ & $-0.0040(2)$ & $-0.00043(6)$ & $-0.0024(6)$ \\
$b_{1}$ & $0.061(5)$ & $0.066(2)$ & $0.097(4)$ & $0.093(2)$ & $0.205(2)$ \\
$b_{2}$ & $0.14(2)$ & & & $0.018(6)$ & $-0.118(3)$ \\
\hline \hline
\end{tabular}

In the language of renormalization, the finite-size dependence of the singular part of the free energy density $f$ is formulated as

$$
f(t, h, v, \ldots ; L)=L^{-d} f\left(t L^{y_{t}}, h L^{y_{h}}, v L^{y_{i}}, \ldots ; 1\right),
$$

where $t$ is the transverse field, $h$ is the magnetic field, $v$ is the irrelevant field; $y_{t}, y_{h}$, and $y_{i}$ are the corresponding exponents, and $d$ is the dimensionality. Therefore, one expects the following finite-size behavior of $Q_{L}(t)$ near critical point [17],

$$
\begin{aligned}
Q_{L}(t)= & Q+a_{1}\left(t-t_{c}\right) L^{y_{t}}+a_{2}\left(t-t_{c}\right)^{2} L^{2 y_{t}}+\cdots \\
& +b_{1} L^{y_{i}}+b_{2} L^{y_{2}}+c_{1} L^{y_{3}}\left(t-t_{c}\right)+\cdots,
\end{aligned}
$$

where $y_{2}=d-2 y_{h}, y_{3}=y_{i}+y_{t}$, and $a_{1}, a_{2}, b_{1}, b_{2}$, and $c_{1}$ are unknown parameters. The Monte Carlo data were fitted on the basis of this formula, according to the least-squares criterion. The exponents $y_{t}, y_{h}$, and $y_{i}$ were set to the exact Ising values $1,15 / 8$, and -2 , respectively. So $y_{2}=d-2 y_{h}$ $=-7 / 4$ and $y_{3}=y_{i}+y_{t}=-1$. A fit including corrections with amplitudes $a_{1}, a_{2}, a_{3}, a_{4}, b_{1}$, and $c_{1}$ shows that $t_{c}$ $=0.99998(6)$ and $Q=0.80976(22)$, in a good agreement with the known results $t_{c}=1$ and $Q=0.809678(3)$ [16]. When we set $t_{c}=1$ and $Q=0.809678$, and leave $y_{t}$ to be fitted, we obtain $y_{t}=1.01(1)$, in agreement with the known universal properties of the isotropic Ising model.

In order to compare the efficiency of the CWA with the conventional Wolff method, we investigated the $d=2$ TIM on a $L \times L$ square lattice with periodic boundary conditions. The CWA was applied to simulate for the model defined by Eqs. (10) and (13) at the continuous limit $\epsilon=0$. The length of the third direction is taken as $L$. The conventional Wolff algorithm was used to study such anisotropic lattice models with small but nonzero $\epsilon(1 / 150 \leqslant \epsilon \leqslant 1 / 6)$. The lattice size in the $z$ direction is taken as $L / \epsilon$, and the couplings $K_{x y}$ and $K_{z}$ are obtained by substituting $\epsilon$ in Eq. (13). The transverse field was set as $t=3.04440$, which is very close to the critical point $t_{c}$ (see Table I), and the system size at $L=4$.

For several values of $\epsilon$, the computer time needed for 5 $\times 10^{6}$ conventional Wolff steps, was compared to the time needed by the CWA for the same number of steps (see Fig. 3 ). As expected, the efficiency of the conventional Wolff method decreases proportionally for $\epsilon \longrightarrow 0$. The efficiency of the CWA is lower than but still comparable to that of the conventional Wolff method for the isotropic Ising model. The reasons are as follows: first, floating-point operations are necessary in the anisotropic limit since the third dimension is continuous; and second, to find the sign at a particular position, one has to find the number of interfaces between this position and the origin of the line where it is located. The time consumption to find the signs is however still acceptable for the system sizes used in this work.

During these simulations, also the quantity $Q_{L}(\epsilon)$ was sampled as a function of $\epsilon$. The results obtained by the conventional Wolff method as $\epsilon \rightarrow 0$, display satisfactory convergence to $Q_{L}(0)$ as obtained by the CWA (see Fig. 4). The convergence takes place approximately as $\epsilon^{2}$. The data for $Q_{L}(\epsilon)$ were fitted according to the least-squares criterion by the formula

$$
Q_{L}(\epsilon)=Q_{L}(0)+q_{1} \epsilon+q_{2} \epsilon^{2}+q_{3} \epsilon^{3}+\cdots,
$$

where $q_{1}, q_{2}$, and $q_{3}$ are unknown parameters. The largest $\epsilon$ included in the fit is 0.4 , and we also let $Q_{L}(0)$ to be fitted. The resulting fit satisfied the $\chi^{2}$ criterion $\left(\chi^{2}=18\right.$ for 17 degrees of freedom); we obtain $q_{1}=-0.0006(8), q_{2}=$ $-0.1322(5), q_{3}=-0.0076(10)$, and $Q_{L}(0)=0.65194(7)$, which is consistent with the Monte Carlo data obtained by

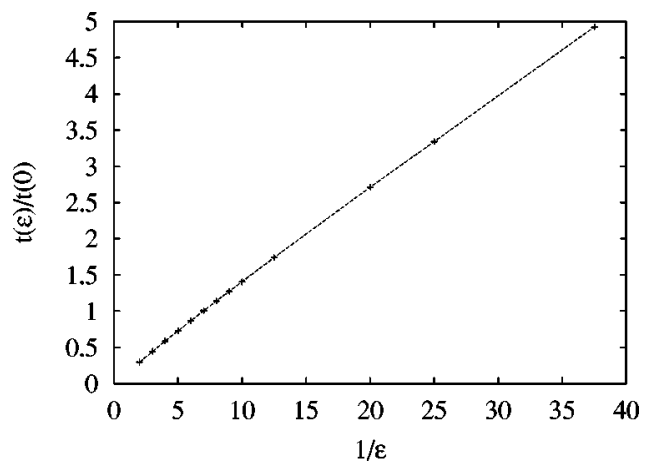

FIG. 3. Ratio between the time used by the conventional Wolff algorithm and that by the present continuous algorithm, as a function of $1 / \epsilon .5 \times 10^{6}$ Wolff steps are taken for every simulation. The system size and the transverse field are $L=4$ and $t=3.04440$, respectively. 


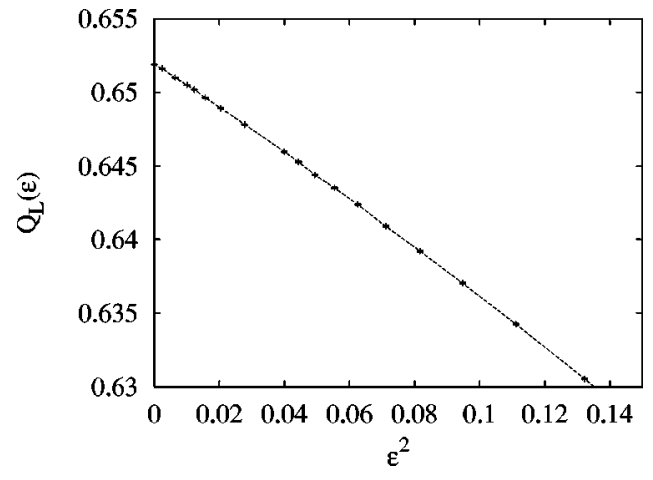

FIG. 4. Dependence of $Q_{L}(\epsilon)$ on $\epsilon$, as a function of $\epsilon^{2}$, for a system with sizes $L \times L / \epsilon$, with $L=4$. The transverse field $t$ $=3.04440$ is set near its critical value. Error bars are approximately equal to the thickness of the lines.

the CWA $Q_{L}(0)=0.65188(6)$. This fit suggests that the term with the amplitude $q_{1}$ vanishes.

This can be explained by Eq. (13). Since the critical points $t_{c}(\epsilon)$ for nonzero $\epsilon$ is expected to deviate from $t_{c}(0)$ as $\epsilon^{2}$, while the transverse field was always set as $t_{c}(0)$ during the simulations, it is not surprising that $Q_{L}(\epsilon)$ $-Q_{L}(0) \propto \epsilon^{2}$.

\section{APPLICATIONS}

\section{A. Critical points of several TIM's}

By means of the CWA, we have investigated the $d=2$ TIM's on the square, triangular, honeycomb, and Kagome lattices (see Fig. 5), and the $d=3$ TIM on the simple-cubic lattice. System sizes were chosen as $L^{d}$, so that the physical length $\epsilon M$ of the continuous direction is equal to $L$. Periodic boundary conditions were imposed. During the simulations, the Binder parameter $Q_{L}(t)$ was sampled.

For the $d=2$ TIM's, the finite-size behavior of $Q_{L}(t)$ is expected to follow the usual scaling behavior of threedimensional Ising system as expressed by Eq. (23). Moreover, we expect that the $d=2$ TIM's on different lattices share the same exponents for the scaling fields $t, h$, and $v$, although the unknown amplitudes can be different. The Monte Carlo data for the systems on these different lattices were independently fitted on the basis of Eq. (23), according to the least-squares criterion. The exponents $y_{t}$ and $y_{i}$ are set to the known values $1.587(2)$ and $-0.815(4)$, respectively, as reported in the literature, for instance, Refs. [18-20] and in papers referenced therein. Results of these fits are shown in Table I.

To test the universality of the $d=2$ TIM's, we set $Q$ $=0.6206$ and $t_{c}=3.04438$ as in Table I for the square lattice, and thus obtained $y_{t}=1.583(6)$, which is in agreement with the known value 1.587. The corrections with amplitudes $a_{1}$, $a_{2}, a_{3}, a_{4}, b_{1}$, and $b_{2}$ were included, and the smallest system size used in the fit is $L=2$.

For the $d=3$ TIM, we have to deal with the numerical difficulties associated with the corrections due to the marginally irrelevant field as occur in four-dimensional Ising-like models $[21,22]$. The anomalously slow renormalization flow near the fixed point translates into a similarly slow finite-size convergence of the Binder ratio $Q_{L}(t)$, and leads to correction factors including small powers of logarithms of the linear system size $L$. Under these circumstances it is not feasible to determine many independent parameters in the fit. We thus make use of the theoretical predictions for the universal value $Q$ at the critical point and the values of the exponents of the scaling fields. Expanding the finite-size scaling function for $Q_{L}(t)$, we expect the following behavior [22]:

$$
\begin{aligned}
Q_{L}(t)= & Q+\sum_{k} a_{k}\left\{L^{y_{t}}(\ln L)^{\zeta_{t}}\left[t-t_{c}+v \frac{L^{-y_{t}}}{(\ln L)^{2 / 3}}\right]\right\}^{k} \\
& \times b_{1} L^{y_{2}}+b_{2}(\ln L)^{-1}+b_{3}(\ln L)^{-2} \cdots,
\end{aligned}
$$

where $k=1,2, \ldots, \zeta_{t}=\frac{1}{6}, y_{t}=2$, and $y_{2}=4-2 y_{h}=-2$. The "shift" term with amplitude $v$ seems unimportant, and was taken to be zero. The universal value $Q$ is taken as the analytical value: $Q=0.456947[21,23]$. Results are shown in Table I.

Thus, by applying the CWA to these two- or threedimensional TIM's, we obtained the critical values of the transverse fields $t_{c}$ (see Table I). Table II compares these values to those obtained by other methods which include effective-field approximation [24,25], effective-field renormalization group (EFRG) [26,27], series expansion [28,29], and density-matrix renormalization [31] results. The precision of the present results indicates that our algorithm contributes a useful tool for numerical studies of transverse Ising models. The total computer time consumed by the present simulations is about 5 processor-months at $750 \mathrm{MHz}$.

\section{B. The Binder ratio and the determination of the length scale}

For the isotropic Ising model on the simple-cubic lattice, i.e., $K_{x y}=K_{z}$ in Eq. (10), the ratio $Q$ defined by Eq. (23) is a universal function $Q(\alpha)$ of the aspect ratio $\alpha=M / N$. On the basis of symmetry arguments it is plausible that an extremum occurs at $\alpha=1$.

For the corresponding anisotropic system, as obtained by extending the TIM on the square lattice in the Trotter direction, we expect a different dependence of the Binder ratio
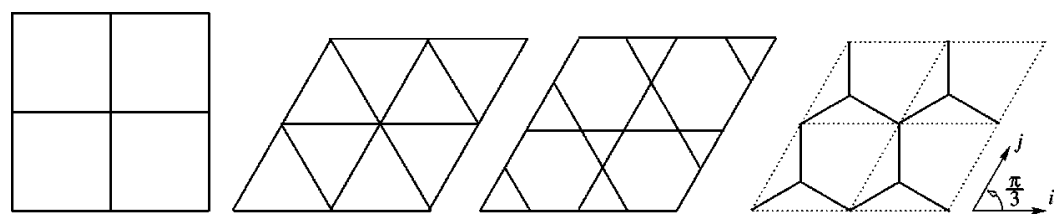

FIG. 5. The two-dimensional lattices on which the TIM's are investigated. From left to right: square, triangular, Kagome, and honeycomb lattices with finite size $2 \times 2$. The geometries of the corresponding anisotropic limit of the classical Ising models are parallel lines which originate from these lattice sites and perpendicular to the planes. 
TABLE II. Comparison of the critical values of the transverse field $t_{c}$, as obtained by several different methods, for the $d=2$ TIM's on the square, Kagome, honeycomb, and triangular lattices, and the $d=3$ TIM in the simple-cubic lattice.

\begin{tabular}{lccccccc}
\hline \hline Lattice & $\begin{array}{c}\text { Present } \\
\text { work }\end{array}$ & $\begin{array}{c}\mathrm{EFA}^{\mathrm{a}} \\
{[24,25]}\end{array}$ & $\begin{array}{c}\mathrm{EFRG}^{\mathrm{b}} \\
{[27]}\end{array}$ & $\begin{array}{c}\mathrm{SE}^{\mathrm{c}} \\
{[28,29]}\end{array}$ & $\begin{array}{c}\mathrm{PI}^{\mathrm{d}} \\
{[6]}\end{array}$ & $\begin{array}{c}\mathrm{S}^{2} \mathrm{~W}^{\mathrm{e}} \\
{[30]}\end{array}$ & $\begin{array}{c}\mathrm{DMRG}^{\mathrm{f}} \\
{[31]}\end{array}$ \\
\hline Square & $3.04438(2)$ & 2.742 & 3.021 & 3.08 & 3.225 & $3.044(1)$ & 3.046 \\
Kagome & $2.95265(4)$ & 2.742 & 2.333 & & & & \\
Honeycomb & $2.13250(4)$ & & & & & & \\
Triangular & $4.76811(9)$ & 4.704 & 4.200 & 4.118 & & & \\
Cubic & $5.15813(6)$ & 4.704 & 5.059 & 5.153 & & & \\
\hline \hline
\end{tabular}

${ }^{\mathrm{a}}$ Effective field approximation.

${ }^{\mathrm{b}}$ Effective field renormalization group.

${ }^{\mathrm{c}}$ Series expansion.

${ }^{\mathrm{d}}$ Path integral Monte Carlo simulation.

${ }^{\mathrm{e}} \mathrm{S}$ wendsen-Wang in continuous time.

${ }^{\mathrm{f}}$ Density matrix renormalization group.

$Q_{a}(\alpha)$ on the aspect ratio $\alpha=M_{p} / N$, where $M_{p}=\epsilon M$ is the physical size in the continuous dimension. This can be attributed to the spatial anisotropy of the Hamiltonian density at the fixed point of the anisotropic Ising model. However, the anisotropy of the fixed-point Hamiltonian can be suppressed by an anisotropic rescaling in the strong-coupling direction, i.e., $z \rightarrow z^{\prime}=\beta z$ so that $0<z^{\prime} \leqslant \beta M_{p}$. Thus we expect that $Q_{a}(\alpha)$ of the anisotropic Ising model follows the same universal function $Q$, but with $\alpha$ replaced by $\alpha^{\prime}=\beta \alpha$ $=\beta M_{p} / N$, i.e., $Q_{a}(\alpha)=Q(\alpha \beta)$. Using instead the logarithm of the aspect ratio as the independent variable, one finds that $Q_{a}(\ln \alpha)=Q(\ln \alpha+\ln \beta)$, which expresses a shift on the $\ln \alpha$ scale with respect to the isotropic case.

We determined $Q_{a}(\ln \alpha)$ by means of Monte Carlo simulations for system sizes $L \times L \times \alpha L$, with $L=8,12,16,20$, and 24, and several values of $\alpha$ in the range from 0.3 to 3.0. These data were analyzed on the basis of the scaling formula

$$
\begin{aligned}
Q_{L}(\ln \alpha+\ln \beta)= & Q_{\infty}(0)+v_{i} L^{y_{i}}+v_{2} L^{y_{2}}+\left(1+d L^{y_{i}}\right) \\
& \times \sum_{k \geqslant 2} a_{k}\left(\ln \beta+\ln \alpha+c L^{y_{a}}\right)^{k},
\end{aligned}
$$

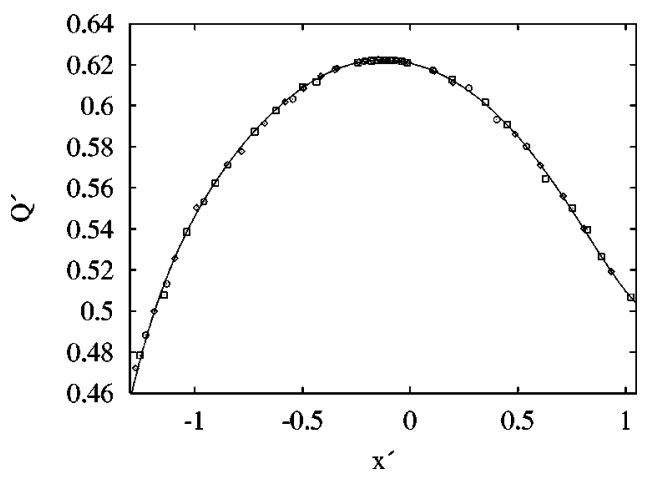

FIG. 6. Data collapse of $Q_{L}(\ln \alpha+\ln \beta)$ shown as $Q^{\prime}=Q_{L}$ $-v i L^{y_{i}-v_{1} L^{y_{1}}}$ vs $x^{\prime}=\ln \alpha-c L^{y_{i}}$. The system sizes are $L$ $=8(\diamond), L=12(\square)$, and $L=16(\bigcirc)$. The solid line is the corresponding fit. obtained by Taylor expansion in the argument of $Q_{L}$ to which a finite-size correction with amplitude $c$ has been added. This term describes a $L$-dependent shift of the maximum of $Q_{L}$. The value of $Q_{\infty}(0)$ is known to be $0.62358(15)$ [19]. Corrections with amplitudes $v_{i}$ and $v_{2}$ describe the finite-size dependence of $Q$ near its maximum. The term with amplitude $d$ describes the influence of the anisotropy on the irrelevant finite-size correction.

Although the finite-size correction with amplitude $c$ is clearly observable, we could not satisfactorily determine the associated exponent $y_{a}$. We have assumed that $y_{a}=y_{i}$ which is consistent with the data. First, we neglected the term with amplitude $d$ and fixed the value of $Q_{\infty}(0)$ at 0.62358 . A reasonable fit is obtained when we include terms up to $k$ $=7$ in the expansion. We then find $\beta=0.880(6)$, which is slightly lower than the value $\beta=0.8881$ (2) which was obtained from the spin-spin correlation function and quoted in Refs. $[9,20]$. A reasonable data collapse of the numerical finite-size data for $Q$ is thus obtained in the $Q_{L}^{\prime}=Q_{L}$ $-v_{i} L^{y_{i}}-v_{2} L^{y_{2}}$ versus $x^{\prime}=\ln \alpha-c L^{y_{i}}$ diagram, shown in Fig. 6 . However, when we include the term with amplitude $d$ in Eq. (26), we obtain a more satisfactory (on the basis of the $\chi^{2}$ criterion) fit yielding $\beta=0.886(7)$.

In conclusion, the precision of the present results shows that the CWA is an efficient tool for the investigation of quantum models such as the TIM. The results of the data analysis agree with the general belief that the Hamiltonian limit of the Ising model belongs to the same universality class as the isotropic lattice Ising model. Furthermore, the special feature that one of the dimensions is continuous, makes the CWA suitable for applications to models defined in curved geometries [9].

\section{ACKNOWLEDGMENTS}

This research was supported by the Dutch FOM foundation ("Stichting voor Fundamenteel Onderzoek der Materie") which was financially supported by the NWO ("Nederlandse Organisatie voor Wetenschappelijk Onderzoek"). 
[1] T.D. Schultz, and D.C. Mattis, Rev. Mod. Phys. 36, 856 (1964).

[2] M. Suzuki, Prog. Theor. Phys. 46, 1337 (1971).

[3] M. Suzuki, Prog. Theor. Phys. 56, 2454 (1976).

[4] H.F. Trotter, Proc. Am. Math. Soc. 10, 545 (1959).

[5] R.P. Feynman, Statistical Mechanics (Benjamin, Reading, MA, 1972).

[6] R.M. Stratt, Phys. Rev. B 33, 1921 (1986).

[7] A. Wiesler, Phys. Lett. 89A, 352 (1982).

[8] A continuous time algorithm for discrete quantum systems is due to B.B. Beard and U.-J. Wiese, Phys. Rev. Lett. 77, 5130 (1996); the present algorithm for the Ising model was presented by Henk W.J. Blöte at the Statphys 20 Conference, in Livre des Résumés, edited by A. Gervois, M. Gingold, and D. Iagolnitzer (UNESCO Sorbonne, Paris, 1998). A related algorithm of the Swendsen-Wang type was described in Ref. [30].

[9] Y.J. Deng and H.W.J. Blöte, Phys. Rev. Lett. 88, 190602 (2002).

[10] L. Onsager, Phys. Rev. 65, 117 (1944).

[11] A.A. Migdal, Zh. Éksp. Teor. Fiz. 69, 1457 (1975) [Sov. Phys. JETP 42, 743 (1976)].

[12] R.H. Swendsen and J.-S. Wang, Phys. Rev. Lett. 58, 86 (1987); J.-S. Wang and R.H. Swendsen, Physica A 167, 565 (1990).

[13] U. Wolff, Phys. Rev. Lett. 62, 361 (1989); Phys. Lett. B 228, 379 (1989).
[14] E. Luijten and H.W.J. Blöte, Int. J. Mod. Phys. C 6, 359 (1995).

[15] K. Binder, Z. Phys. B 43, 119 (1981).

[16] G. Kamieniarz and H.W.J. Blöte, J. Phys. A 26, 201 (1992).

[17] H.W.J. Blöte, E. Luijten, and J.R. Heringa, J. Phys. A 28, 6289 (1995).

[18] R. Guida and J. Zinn-Justin, J. Phys. A 31, 8103 (1998).

[19] H.W.J. Blöte, L.N. Schur, and A.L. Talapov, Int. J. Mod. Phys. C 10, 1137 (1999).

[20] Y.J. Deng and H. W. J. Blöte (unpublished).

[21] E. Luijten, Interaction Range, Universality and the Upper Critical Dimension (Delft University Press, Delft, 1997).

[22] J.R. Heringa, H.W.J. Blöte, and E. Luijten, J. Phys. A 33, 2929 (2000).

[23] E. Brézin and J. Zinn-Justin, Nucl. Phys. B 257, 867 (1985).

[24] E.F. Samento and T. Kaneyoshi, J. Phys. C 21, 3933 (1988).

[25] P.A. Slotte, J. Phys. A 20, L177 (1987).

[26] Q. Jiang and Z.Y. Li, Phys. Rev. B 40, 11264 (1989).

[27] Q. Jiang and X.F. Jiang, Phys. Lett. A 224, 196 (1997).

[28] R.J. Elliott and A.P. Young, Ferroelectrics 7, 23 (1974).

[29] W.H. Zheng, J. Oitmaa, and C.J. Hamer, J. Phys. A 27, 5425 (1994).

[30] R. Rieger and N. Kawashima, Eur. Phys. J. B 9, 233 (1999).

[31] M.S.L. du Croo de Jongh and J.M.J. van Leeuwen, Phys. Rev. B 57, 8494 (1998). 